\title{
IRREDUCIBLE WAVELET REPRESENTATIONS AND ERGODIC AUTOMORPHISMS ON SOLENOIDS
}

\author{
Dorin ERvin Dutkay, David R. LARson And Sergei Silvestrov
}

\begin{abstract}
We focus on the irreducibility of wavelet representations. We present some connections between the following notions: covariant wavelet representations, ergodic shifts on solenoids, fixed points of transfer (Ruelle) operators and solutions of refinement equations. We investigate the irreducibility of the wavelet representations, in particular the representation associated to the Cantor set, introduced in [13], and we present several equivalent formulations of the problem.
\end{abstract}

Mathematics subject classification (2010): 42C40, 28D05, 47A67, 28A80.

Keywords and phrases: Representation, ergodic automorphism, Cantor set, Ruelle operator, solenoid, refinable function.

\section{REFERENCES}

[1] L.W. BAggett, J. Courter, AND K. Merrill, The construction of wavelets from generalized conjugate mirror filters in $L^{2}\left(\mathbb{R}^{n}\right)$, Appl. Comput. Harmon. Anal., 13 (2002), 201-223.

[2] Ola Bratteli, David E. Evans, and Palle E. T. Jorgensen, Compactly supported wavelets and representations of the Cuntz relations, Appl. Comput. Harmon. Anal., 8, 2 (2000), 166-196.

[3] L.W. Baggett, V. Furst, K.D. Merrill, And J. A. PaCker, Classification of generalized multiresolution analyses, Preprint, 2009.

[4] L.W. Baggett, V. Furst, K.D. Merrill, And J. A. Packer, Generalized low-pass filters and multiresolution analyses, J. Funct. Analysis, 2009.

[5] Berndt Brenken and Palle E. T. Jorgensen, A family of dilation crossed product algebras, J. Operator Theory, 25, 2 (1991), 299-308.

[6] Ola Bratteli and PAlle E. T. JoRgensen, Iterated function systems and permutation representations of the Cuntz algebra, Mem. Amer. Math. Soc., 139, 663 (1999), x+89.

[7] L.W. Baggett, N.S. Larsen, K.D. Merrill, J.A. Packer, and I. Raeburn, Generalized multiresolution analyses with given multiplicity functions, J. Fourier Anal. Appl, 2008.

[8] L.W. Baggett, N.S. Larsen, J.A. Packer, I. Raeburn, And A Ramsay, Direct limits, MRA's and wavelets, Preprint, 2009.

[9] L.W. Baggett, H. Medina, And K. Merrill, Generalized multiresolution analyses, and a construction method for all wavelet sets in $\mathbb{R}^{n}$, J. Fourier Anal. Appl., 5 (1999), 563-573.

[10] Alain Connes, Noncommutative geometry, Academic Press Inc., San Diego, CA, 1994.

[11] JeAn-Pierre Conze AND Albert RAUgi, Fonctions harmoniques pour un opérateur de transition et applications, Bull. Soc. Math. France, 1183 (1990), 273-310.

[12] IngRID DAUbeCHIES, Ten lectures on wavelets, volume 61 of CBMS-NSF Regional Conference Series in Applied Mathematics, Society for Industrial and Applied Mathematics (SIAM), Philadelphia, PA, 1992.

[13] Dorin E. Dutkay and Palle E. T. Jorgensen, Wavelets on fractals, Rev. Mat. Iberoam., 22, 1 (2006), 131-180.

[14] Dorin Ervin DutKay and Palle E. T. Jorgensen, Hilbert spaces built on a similarity and on dynamical renormalization, J. Math. Phys., 47, 5 (2006), 053504, 20.

[15] Dorin Ervin Dutkay and Palle E. T. Jorgensen, Martingales, endomorphisms, and covariant systems of operators in Hilbert space, J. Operator Theory, 58, 2 (2007), 269-310. 
[16] Xingde Dai And David R. Larson, Wandering vectors for unitary systems and orthogonal wavelets, Mem. Amer. Math. Soc., 134, 640 (1998), viii+68.

[17] D. E. DutKay AND S. Silvestrov, Reducibility of the wavelet representation associated to the Cantor set, preprint, 2010, http://arxiv.org/abs/1008.4349

[18] Kenneth Falconer, Fractal geometry, John Wiley \& Sons Inc., Hoboken, NJ, second edition, 2003. Mathematical foundations and applications.

[19] Alex Furman, Orbit equivalence rigidity, Ann. of Math. (2), 150, 3 (1999), 1083-1108.

[20] Richard F. Gundy, Low-pass filters, martingales, and multiresolution analyses, Appl. Comput. Harmon. Anal., 9, 2 (2000), 204-219.

[21] Richard F. GUNDY, Probability, ergodic theory, and low-pass filters, In Topics in harmonic analysis and ergodic theory, volume 444 of Contemp. Math., pages 53-87. Amer. Math. Soc., Providence, RI, 2007.

[22] Deguang Han and David R. Larson, Frames, bases and group representations, Mem. Amer. Math. Soc., 147, 697 (2000), x+94.

[23] John E. Hutchinson, Fractals and self-similarity, Indiana Univ. Math. J., 30, 5 (1981), 713-747.

[24] Jun TOMiYAma, The interplay between topological dynamics and theory of $C^{*}$-algebras, volume 2 of Lecture Notes Series, Seoul National University Research Institute of Mathematics Global Analysis Research Center, Seoul, 1992. 